ARTICLE

https://doi.org/10.1038/s41467-018-08241-3

\title{
A diversity-oriented rhodamine library for wide- spectrum bactericidal agents with low inducible resistance against resistant pathogens
}

Xiao Luo ${ }^{1,2}$, Liujia Qian ${ }^{3,4}$, Yansheng Xiao², Yao Tang², Yang Zhao5, Xia Wang5, Luyan Gu², Zuhai Lei², Jianming Bao ${ }^{4}$, Jiahui $\mathrm{Wu}^{4}$, Tingting $\mathrm{He}^{2}$, Fupin $\mathrm{Hu}^{6}$, Jing Zheng ${ }^{5}$, Honglin $\mathrm{Li}^{5}$, Weiping Zhu², Lei Shao ${ }^{4}$, Xiaojing Dong ${ }^{4}$, Daijie Chen ${ }^{3,4}$, Xuhong Qian ${ }^{1,2}$ \& Youjun Yang (i) ${ }^{1,2}$

Antimicrobial resistance is a public health emergency and warrants coordinated global efforts. Challenge is that no alternative molecular platform has been identified for discovery of abundant antimicrobial hit compounds. Xanthene libraries have been screened for bioactive compounds. However, the potentially accessible chemistry space of xanthene dyes is limited by the existing xanthene synthesis. Herein we report a mild one-step synthesis, which permits late-stage introduction of a xanthene moiety onto i.e. natural products, pharmaceuticals, and bioactive compounds and construction of a focused library of rhodamine dyes exhibiting facile functional, topographical and stereochemical diversity. In vitro screening yields 37 analogs with mid-to-high bactericidal activity against WHO priority drugresistant pathogens. These findings suggest that synthetic dye libraries exhibiting high structural diversity is a feasible chemical space combating antibacterial resistance, to complement the natural sources.

\footnotetext{
${ }^{1}$ State Key Laboratory of Bioreactor Engineering, East China University of Science and Technology, Shanghai 200237, China. ${ }^{2}$ Shanghai Key Laboratory of Chemical Biology, School of Pharmacy, East China University of Science and Technology, Shanghai 200237, China. ${ }^{3}$ School of Pharmacy, Shanghai Jiao Tong University, Shanghai 200240, China. ${ }^{4}$ State Key Laboratory of New Drug and Pharmaceutical Process, Shanghai Institute of Pharmaceutical Industry, Shanghai 201203, China. ${ }^{5}$ Shanghai Key Laboratory of New Drug Design, School of Pharmacy, East China University of Science and Technology, Shanghai 200237, China. ${ }^{6}$ Institute of Antibiotics, Huashan Hospital, Fudan University, Shanghai 200040, China. These authors contributed equally: X.L., L.J.Q. Correspondence and requests for materials should be addressed to Y.Y. (email: youjunyang@ecust.edu.cn) or to D.C. (email: hccb001@163.com) or to X.Q. (email: xhqian@ecust.edu.cn)
} 
A ntibiotics have revolutionized medical practices, have dramatically increased the average life expectancy of humans and are among the greatest medical breakthroughs of the last century. However, antibiotics have only a limited period of clinical-utility before offset by the inevitable emergence of resistance ${ }^{1}$. For example, it took only a few years before resistance toward many antibiotics was discovered, such as penicillin, gentamicin, and ceftazidime ${ }^{2}$. Vancomycin lasted longer before resistance emerged, partly because it was typically used as the last resort. Nevertheless, vancomycin intermediate/ resistant pathogens are now widespread ${ }^{3}$. New antibiotics are desperately sought after to fight bacterial resistance 4 . Ironically, large pharmaceutical companies have dramatically shrunk or cut their antibiotic programs. The reasons for this situation are manifold. The golden mine for antibiotics, the secondary metabolites of soil actinomycetes, has run dry after decades of exploitation $^{5,6}$. An alternative chemical space for abundant antibiotic hit compounds is yet to be identified ${ }^{7}$. Third, antibiotic development has a low expected profit return. As a result, the antibiotic pipeline has been running at an alarmingly slow pace in the past few decades ${ }^{8}$. In particular, among these recently approved few antibiotics, most are actually chemically modified derivatives of the existing classes of drugs, most of which are of natural origin ${ }^{9-12}$. The resistant strains may readily mutate to resist these analogs if their existing resistance mechanisms do not already exhibit partial cross-effectiveness ${ }^{13-16}$. The genes encoding resistance to the natural products that are present within the original organisms can also be horizontally transferred to pathogenic microbes enabling resistance to emerge. To prevent the post-antibiotic era becoming reality, alternative chemical space capable of producing abundant antibiotic scaffolds are warranted.

Previously untapped natural sources, i.e. uncultured soil microbes ${ }^{17}$, ocean bacteria ${ }^{18}$, unexpressed metabolites coded by silent operons ${ }^{19}$, and human commensals ${ }^{20}$, have recently been explored and yielded antibiotic compounds. At the same time, synthetic libraries should not be overlooked for antibiotic discovery $^{4}$. Salvarsan for syphilis and prontosil for streptococcus were two classic antimicrobial drugs discovered from the synthetic dye libraries in the early 20th century, and other notable examples are quinolones and the recent addition of oxazolidinones ${ }^{21}$. Despite the fact that target-based antibiotic discovery since the 1990 s has been largely unsuccessful ${ }^{7,22}$, synthetic library of unique chemical space exhibiting high structural complexity and diversity offers opportunities for formulating new paradigms for antibiotic development ${ }^{23-25}$. Recently, the diversity-oriented fluorescence library approach (DOFLA) has been proven to be a robust method for discovery of bioactive molecules via phenotypic screening ${ }^{26}$. Xanthene dyes, classic small-molecule fluorophores including fluorescein, rhodamine and rhodol, has recently attracted attentions for DOFLA. For example, Chang et al. and other research groups constructed a number of focused libraries based on various fluorescent scaffolds ${ }^{27,28}$, and further discovered useful imaging agents or fluorescent probes for smallmolecule targets, nucleic acids, proteins, lipid droplets, stem-cells and live animals ${ }^{29-31}$. Burgess et al. constructed a library of mitochondria-targeting rhodamine analogues exhibiting potent anti-proliferative effects toward tumor cells ${ }^{32,33}$. High structural diversity of dye libraries, the key to its success in the fluorescence library approach, is however the biggest obstacle to overcome. The ubiquitous structure feature of a dye is a two-dimensional conjugated system, to which some simple chemical groups may be attached. Therefore, dyes are typically not endowed with complex three-dimensional features and not ideal for discovery of bioactive compounds via phenotypic screening. Also, the conditions for dye synthesis are usually harsh and installation of chemical groups exhibiting exotic functional, topological and stereochemical features are difficult ${ }^{34-36}$. Diversity-oriented rhodamine library could not be constructed with the conventional method due to, extensive side-reactions, tedious purification, low yields and most important of all limited scope of compatible substrates. Take the xanthene dye library by Ahn et al. ${ }^{37}$ as an example. It is a chemical library of $300+$ compounds. However, all follow the general feature, i.e. a xanthene core with a small aromatic group at its C-9. Therefore, the library is not high in terms of diversity. A diversity index of only 3.228 was calculated for this library of rhodamine dyes with Extended-Connectivity FingerPrint (ECFP) ${ }^{38-40}$, which is a convenient estimate of the structural diversity of a chemical library (vide infra).

We herein report the construction of a diversity-oriented rhodamine library and discovery of potent and wide-spectrum bactericidal agents without inducible resistance. Phenotypic screening of the library yields two promising hit compounds, and RD22 and RD53 were found to be particularly noteworthy through further studies. They inhibit the growth of a widespectrum of pathogens, with a potent MIC of $0.5-2 \mu \mathrm{g} \mathrm{mL}-1$ against the Gram-positive strains, and a MIC of $2-16 \mu \mathrm{g} \mathrm{mL}-1$ against the Gram-negative strains. They are potent bactericidal agents and pathogens do not readily acquire resistance by genomic mutations toward RD22 and RD53. They exhibit low haemolytic activity with a Lysis 20 over $100 \mu \mathrm{g} \mathrm{mL} \mathrm{m}^{-1}$. The aforementioned properties of RD22 and RD53, along with other less potent analogs suggest that the rhodamine library is a viable source of antibacterial hit compounds.

\section{Results}

Construction of a library with 70 rhodamine analogs. A number of preparative methods are available for xanthene dyes. The earliest synthesis was by von Baeyer ${ }^{34}$ in 1871 through the condensation of phthalic anhydride with phenol at high temperature, with Lewis acid or strong proton acid as the catalyst. To accommodate the growing demands for functional xanthene dyes, an alternative mild preparation was introduced by Tsien et al. in 1989, involving nucleophilic addition of a phenyl Grignard (or lithium) reagent with a xanthenone at low temperature ${ }^{36}$. This has enabled the preparation of the library of Ahn et al. ${ }^{37}$. Though robust, diversity of the xanthene dyes from this method is still limited by the availability and structural versatility of Grignard reagents. The limitation remains unsolved with the report of twostep Grignard/demethylation method by Yang et al. ${ }^{41}$. To address those difficulties with the classic xanthene synthesis, we have developed a mild one-step and high-yielding xanthene synthesis (Fig. 1a) via nucleophilic condensation of a dilithium reagent (2) with readily available esters, anhydrides, or even some amides (Supplementary Figure 1). This method is versatile and allows the preparation of xanthene dyes with exotic substituents at C-9, e.g. phenyls, aryls, alkyls, alkenyls and acyls (vide infra) (Fig. 1). Shortly after we filed a Chinese invention patent ${ }^{42}$ of this method in March 2017, Grimm et al. $^{43}$ and Fischer and Sparr ${ }^{44}$ also independently developed and reported this method in recent literature.

The capability of this method in preparation of rhodamines with substituted phenyl group at C-9 was first examined (Fig. 1b). As a proof-of-concept experiment, the dilithium reagent (1) was reacted with an alkyl benzoate, i.e. methyl benzoate (S1), to furnish RD1 in an 83\% yield. Rhodamines with a methyl (RD2), hydroxylmethyl (RD3), formal (RD5) or a carboxyl group (RD6) installed at the ortho-position of the bottom phenyl ring was also smoothly prepared in excellent yields by reacting 1 with 2methyl-benzoate (S2), phthalide (S3), 3-methoxyphthalide (S4) or phthalic anhydride (S6). RD4, rather than RD5, could be 

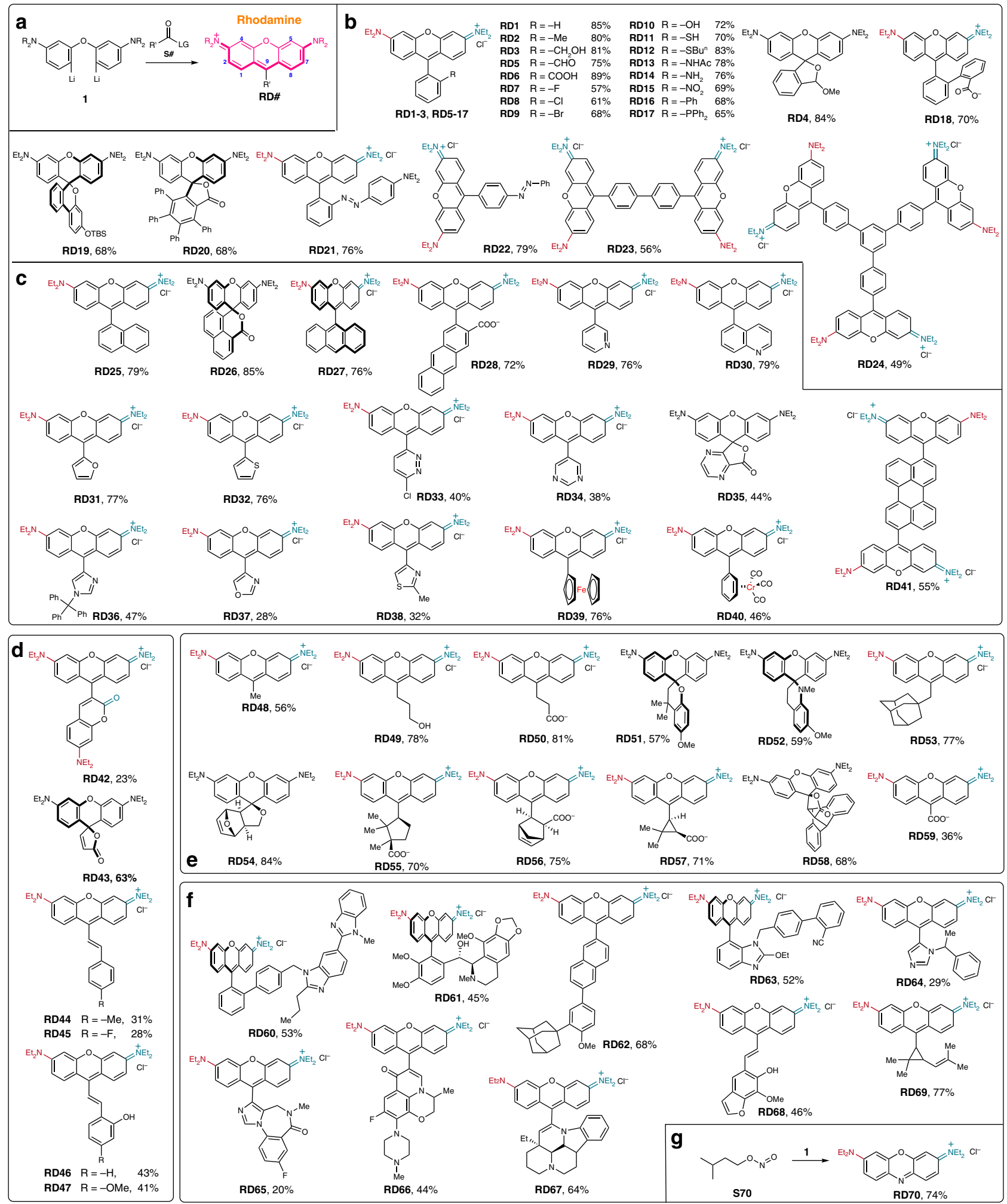

Fig. 1 The rhodamine library via nucleophilic condensation of a dilithium reagent $\mathbf{1}$ with various substrates. a The general synthetic pathway. Structures of rhodamine dyes with b phenyl groups at C-9 (RD1-RD24); c polyaromatic or heteroaryl groups at C-9 (RD25-RD41); d alkenyl groups at C-9 (RD42RD47); e alkyl groups at C-9 (RD48-RD59); $\mathbf{f}$ bioactive molecules at C-9 (RD60-RD69) and $\mathbf{g}$ oxazine (RD70). Most of the rhodamine dyes are in their chloride salt form

obtained in an $84 \%$ yield if the reaction was quenched with saturated $\mathrm{NH}_{4} \mathrm{Cl}$, rather than dilute $\mathrm{HCl}$ solution.

The one-step high-yielding synthesis of RD3 and RD5 shown here is superior to their literature preparations ${ }^{45,46}$. The ortho fluoro-, chloro- or bromo-substituted methyl benzoate (S7-S9) were compatible with this method to give RD7-RD9 in good to excellent yields. However the attempt with methyl 2iodobenzoate was complicated with deiodonation leading to the 
formation of RD1, presumably via metal-halogen exchange. Rhodamines with hydroxyl, sulfhydryl, acetyl, amino and nitro groups (RD10, RD11, RD13, RD14 or RD15) were readily prepared by reacting 1 with benzo[ $[d][1,3]$ dioxin-4-one (S10), benzo[ $d][1,3]$ oxathiin-4-one (S11) or benzo[ $d][1,3]$ oxazin-4-one (S13), methyl-2-nitrobenzoate (S15) respectively. Synthesis of rhodamine dyes with bulky groups at the ortho position of the bottom phenyl ring is not trivial. This method was powerful in this regard. Reactions between the dilithium reagent 1 and S16, S17, S18, S19 and S20 (Supplementary Figure 1) have produced a series of rhodamine dyes with such bulky groups as phenyls in RD16, RD18, RD19 and RD20, and diphenylphosphoryl in RD17, in a yield ranging from 65 to $70 \%$. The carbonyl of $\mathbf{S 1 8}$ is involved in electronic push-pull system and therefore is not very reactive toward nucleophilic attack. Still, S18 was a viable substrate to react with 1 furnishing a rhodamine (RD18) with an electron-rich phenyl ring at the ortho-position, in a 70\% yield. RD20 is a rhodamine modified with four freely rotatable phenyl groups and structurally analogous to an AIE-luminogen ${ }^{47}$, e.g. tetraphenylethene. Its potentials in AIE-based studies warrant further investigations. Rhodamines with phenyl diazo groups (RD21 and RD22) were also successfully prepared in yields over $76 \%$. Unusual structures like RD23 or RD24, expected to exhibit high molar absorptivity and fluorescence brightness were successfully obtained, in good yields of $56 \%$ and $49 \%$, respectively.

Onto the C-9 position of the rhodamine core can be installed aryl groups other than phenyl, e.g. polyaromatic moities, heterocyclic aromatic moieties and the exotic sandwich/halfsandwich complexes (Fig. 1c). Rhodamines with naphthalene (RD25, RD26) or anthracene (RD27, RD28) were prepared from reactions of 1 with the corresponding ester or anhydride in good isolated yields (typically over 75\%). Esters of pyridine, quinoline, furan, thiophene, pyridazine, pyrimidine, pyrazine, imidazole, oxazole, and thiazole (Supplementary Figure 1) could also react with the dilithium reagent $\mathbf{1}$ to prepare the corresponding rhodamine dyes (RD29-RD38). With the methyl ester of ferrocene, we prepared RD39, in which the ferrocene moiety is directly bonded to the C-9 position of the rhodamine core. We further showed that the half-sandwich complex could be directly attached to the rhodamine core (RD40). The potential applications of RD40 in sensing, imaging and catalysis are attractive. Substrates bearing two ester moieties could be used to template multiple rhodamine cores, to give RD41.

Alkenyl groups can also be installed onto the C-9 position of a rhodamine core (Fig. 1d). The double bond in such rhodamine dyes exhibits facile chemistry and may be useful in site-specific labeling ${ }^{48}$, chemosensing of nucleophiles ${ }^{49}$ and reactive oxygen species $^{50}$ and optical switching ${ }^{51}$. With the ester of a coumarin, a rhodamine-coumarin dye pair (RD42) was synthesized as one example of this group of rhodamine dyes. Reactions between the dilithium reagent $\mathbf{1}$ and a range of substrates like maleic anhydride, cinnamates, or coumarins (Supplementary Figure 1) were all capable of furnishing such alkenyl substituted rhodamine dyes, i.e. RD43-RD47. We note that the double bond of RD43 adopts cis-conformation, as suggested by a coupling constant of $5.4 \mathrm{~Hz}$ between the two alkenyl H's. The double bonds in RD4447 are all in trans-conformation, regardless the cis- or transconformation of the double bonds in the corresponding starting material (S44-S47, Supplementary Figure 1).

Alkyl esters, lactones and anhydrides are all feasible substrates for synthesizing rhodamine dyes and diverse range of complex alkyl groups are also conveniently installed to the C-9 of the rhodamine core (RD48-RD59) (Fig. 1e).

Till this point, we have demonstrated that this rhodamine synthesis is convenient, powerful and reliable. The range of substrates compatible for this method is exceedingly broad, including esters, anhydride, lactone, aryl lactam, and potentially more. To further promote the scope of this rhodamine synthesis, we are particularly interested in the possibility of using ester-/ lactone-/anhydride-containing bioactive molecules, e.g. pharmaceuticals and pesticides (S60-S69, Supplementary Figure 1), in rhodamine synthesis. The structures of such compounds are typically complex and there were no attempts to use them in dye synthesis. Though probably unexpected to the field, many of such compounds, e.g. telmisartan methyl ester (S60), noscapine (S61), adapalene (S62), a precursor of candesartan (S63), etomidate (S64), the methyl ester of flumazenil (S65), levofloxacin methyl ester (S66), vinpocetine (S67), methoxsalen (S68), ethyl chrysanthemate (S69) were indeed viable substrates for this rhodamine synthesis (Fig. 1f).

This method is also suitable for preparation of an oxazine dye RD70 (Fig. 1g) from isoamyl nitrite, which differs from its rhodamine analog in that it bears a nitrogen atom rather than a methine carbon.

Diversity index. The extended-connectivity fingerprint (ECFP) method was employed to assay the diversity of our library because of its wide-acceptance in drug discovery and has been integrated into various commercial softwares. ECFPs encode each atom and its molecular environment within a circle with a diameter of varying radius of chemical bonds. Depending on the chosen bond radius, different numbers of structural features having different sizes are produced. In this study, we introduce the definition of the total number of fingerprint features as a quantitative measure of the structural diversity of a library. We calculated the specified fingerprint for each molecule using ECFP_6 and counted the total number of unique fingerprint features collected over all the molecules. Then the Diversity Number Finger Print Features (DNFPF value) was defined as the total number of fingerprint features divided by the number of molecules. A DNFPF value of 18.875 was calculated for the obtained library of rhodamine dyes (RD1-RD69), while a DNFPF value of 3.228 was found for the Ahn's library ${ }^{37}$ of rhodamine dyes (Supplementary Table 6). This verified that the synthetic method presented herein is feasible for construction of a rhodamine library with much enhanced structural diversity.

Fluorescence properties. The fluorescence properties of RD1RD70 were studied predominantly in $\mathrm{PBS}(50 \mathrm{mM}, \mathrm{pH}=7.4$ containing $1 \%$ DMSO) with UV-Vis absorption and fluorescence spectroscopies (Fig. 2 and Supplementary Table 1). Other solvents were not tested because we are typically concerned with the potentials of this library in bio-oriented applications. The maximal absorption wavelengths of rhodamine dyes with alkyl groups (RD48-RD59) at C-9 typically absorb at shorter wavelength region of $545-560 \mathrm{~nm}$ and ones substituted with heteroaryl groups (RD25-RD41) absorb at longer wavelength region of 551-585 nm, while others absorb in the range of $560-570 \mathrm{~nm}$. Most dyes exhibit a small Stokes shift of $c a$. 15-30 nm, which is typical of a rhodamine, some have displayed a Stokes shift larger than $30 \mathrm{~nm}$, e.g. $34 \mathrm{~nm}$ for RD31, $41 \mathrm{~nm}$ for RD33, $37 \mathrm{~nm}$ for RD35, and $34 \mathrm{~nm}$ for RD41, $32 \mathrm{~nm}$ for RD47, $39 \mathrm{~nm}$ for RD53, $35 \mathrm{~nm}$ for RD56, $42 \mathrm{~nm}$ for RD59. The fluorescence brightness of many dyes of this library is comparable or higher than that of the rhodamine B (RD6). They are useful for cell imaging-based applications (Supplementary Figure 72-131). Many others are much less fluorescent. Dim dyes may be used as fluorescent probes, in case their fluorescence may be chemospecifically turned on. For example, we have found that RD17 can respond to nitroxyl (HNO) and induce a ca. 5 fold fluorescence turn-on 


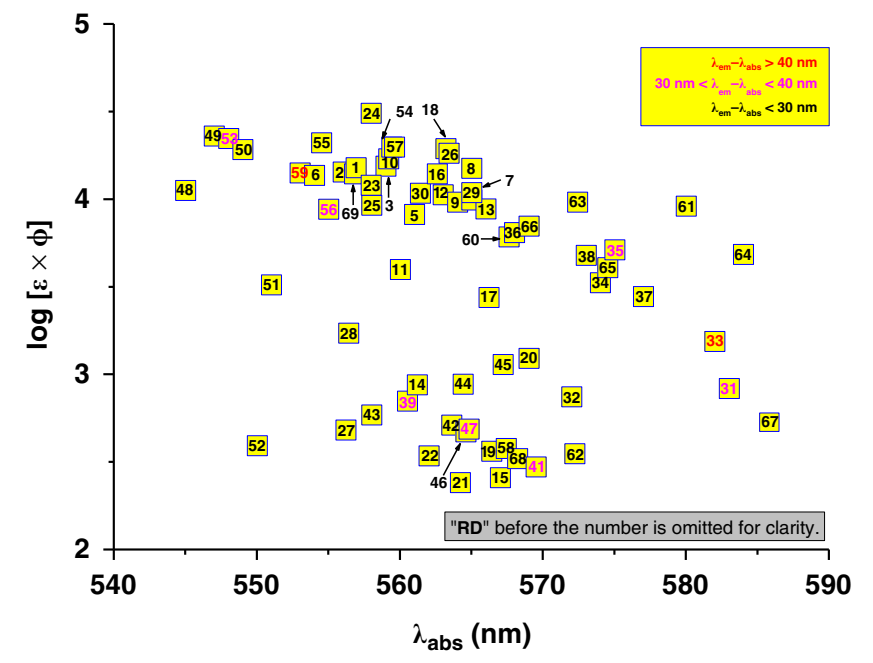

Fig. 2 Fluorescence properties of the rhodamine library in PBS buffer. The $y$ axis is the brightness, which is expressed as $\log (\varepsilon \times \Phi)$ of a given rhodamine dye and the $x$-axis is the absorption maximum. RD23 and RD24 were tested in $\mathrm{EtOH}$

(Supplementary Figure 70) and RD28 may scavenge ${ }^{1} \mathrm{O}_{2}$ and produce a fluorescence turn-on of $c a .14$ fold (Supplementary Figure 71).

Antibacterial studies of the rhodamine library. The antimicrobial activity of (RD1-RD70) were preliminarily evaluated against two drug-resistant bacteria, which were a strain of Grampositive methicillin-resistant Staphylococcus aureus (MRSA, ATCC43300) and a strain of Gram-negative Acinetobacter baumannii (ATCC19606). The minimum inhibitory concentrations (MIC) of each compound were measured using the standard CLSI broth protocol (Fig. 3, Supplementary Table 2). Typically, antimicrobial potency of a compound is considered mid-to-high if its MIC is $8 \mu \mathrm{g} \mathrm{mL}^{-1}$ or lower. Out of the total 70 analogs, 37 were found to exhibit a MIC of $8 \mu \mathrm{g} \mathrm{mL} L^{-1}$ or lower against MRSA, and 5 aganist $A$. baumannii. This is a result much to our delight as well as to our surprise considering the relentless yet largely futile efforts that the community has dedicated to the quest for a molecular platform for discovery of abundant compounds with antimicrobial activity. Further analysis revealed that these bioactive analogs are quite scattered in terms of the nature of substituent at the C-9 position of the rhodamine core. Among these bioactive analogs, 14 analogs bear a phenyl group at C-9 position, 12 a heteroaromatic moiety, 6 an alkenyl and 5 an alkyl group. Presumably, the rhodamine core is the pharmacophore and the substituent at the C-9 playing a subsidiary, yet critical role. These results strongly suggested that this diverse-oriented rhodamine library is a viable platform for discovery of potent antimicrobial agents.

Though all these $37+5$ compounds with mid-to-high antibacterial activity worth further pursuing, we prioritized the ones with a higher activity for in-depth study of their antibiotic spectrum (Table 1). Those compounds with the lowest MIC's against ATCC43300 (RD1/15/22/31/45/46/53) or ATCC19606 (RD12/22/44/45/53) were chosen for further screening against three more Gram-positive bacteria, including the ATCC25923 (methicillin-sensitive Staphylococcus aureus, MSSA), ATCC51299 (vancomycin-resistant Enterococcus faecalis, VRE), and ATCC29212 (vancomycin-sensitive Enterococcus faecalis, VSE), and three Gram-negative bacteria, including ATCC13883 (Klebsiella pneumoniae), ATCC25922 (Escherichia coli) and ATCC27853 (Pseudomonas aeruginosa) (Table 1). RD22/46/53
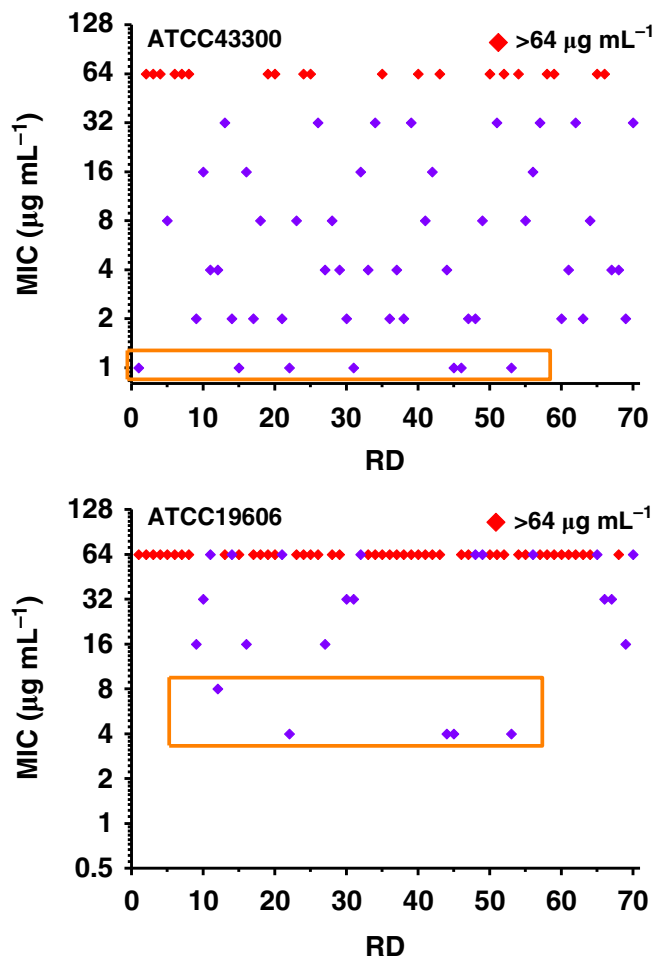

Fig. 3 Activity of RD1-RD70 against MRSA and Acinetobacter baumannii. The MIC ( $\mu \mathrm{g} \mathrm{mL}{ }^{-1}$ ) of each compound was measured by the standard CLSI broth protocol

showed potential broad-spectrum Gram-positive antibiotic activity with a low MIC of $0.5-2 \mu \mathrm{g} \mathrm{mL}{ }^{-1}$, while $\mathbf{R D 1} / \mathbf{1 5} / \mathbf{3 1} / \mathbf{4 5}$ were much less active toward $E$. faecalis compared to their activity against $S$. aureus. RD22/44/53, but not RD12/45, also exhibited antibiotic activity toward Gram-negative bacteria other than ATCC19606, with a relatively higher MIC of $8-32 \mu \mathrm{g} \mathrm{mL}-1$. From this study, RD22/53 stood out as the two most attractive hits. First, their MIC's against MRSA is $1 \mu \mathrm{g} \mathrm{mL} L^{-1}$, the lowest among this shortlist, and comparable or even lower than those of vancomycin and linezolid (Table 1), the last resorts for MRSA infections. Second, they were also equally potent toward both vancomycin-sensitive and vancomycin-resistant $E$. faecalis with a MIC of $2 \mu \mathrm{g} \mathrm{mL} L^{-1}$. Third, they were also active toward various clinically significant Gram-negative bacteria with MIC of 4-16 $\mu \mathrm{g}$ $\mathrm{mL}^{-1}$. These encouraging preliminary results promoted us to explore the antibiotic profile of RD22/53 in a greater depth.

The antibiotic spectrum of RD22 and RD53 were further checked against a panel of standard reference and clinical-isolated Gram-positive and Gram-negative strains, which encompass most of the 12 deadliest drug-resistant bacteria ${ }^{52}$ classified by the World Health Organisation (WHO) (Table 2). The Grampositive strains include S. aureus (MRSA, MSSA), E. faecalis and E. faecium (VRE and VSE), S. epidermidis, S. pyogenes, H. pylori, $S$. pneumonia, while the tested Gram-negative strains are $A$. baumannii, P. aeruginosa, E. coli., K. pneumonia, and S. flexneri. RD53 exhibited a potent MIC of $0.25-2 \mu \mathrm{g} \mathrm{mL}-1$ toward all the aforementioned Gram-positive strains, regardless of the drugresistance profile of these bacteria. The potency of RD53 toward the Gram-negative strains was typically lower compared to the Gram-positive strains. For example, a MIC of $4 \mu \mathrm{g} \mathrm{mL}^{-1}$ was observed for A. baumannii, and $16 \mu \mathrm{g} \mathrm{mL}^{-1}$ for P. aeruginosa, 2-8 $\mu \mathrm{g} \mathrm{mL}^{-1}$ for various strains of $E$. coli, $4-16 \mu \mathrm{g} \mathrm{mL}-1$ for $K$. Pneumoniae and $1 \mu \mathrm{g} \mathrm{mL}^{-1}$ for Shigella flexneri. RD53 was not only found to be effective toward standard reference strains, but 
Table 1 Antimicrobial activity of selected RD dyes against four Gram-positive and four Gram-negative bacteria

\begin{tabular}{|c|c|c|c|c|c|c|c|c|}
\hline MIC ( $\left.\mu \mathrm{g} \mathrm{mL}^{-1}\right)$ & \multicolumn{4}{|c|}{ Gram-positive organism } & \multicolumn{4}{|c|}{ Gram-negative organism } \\
\hline$\overline{R D 1}$ & 1 & 0.5 & 16 & 16 & / & 1 & / & 1 \\
\hline RD15 & 1 & 0.5 & 32 & 16 & / & $/$ & / & $/$ \\
\hline RD22 & 1 & 0.5 & 2 & 2 & 4 & 16 & 16 & 16 \\
\hline RD31 & 0.5 & 0.5 & 16 & 16 & / & / & / & / \\
\hline RD44 & I & / & / & I & 4 & 32 & 8 & 16 \\
\hline RD53 & 1 & 1 & 2 & 2 & 4 & 16 & 8 & 16 \\
\hline vancomycin & 2 & 2 & / & 4 & / & / & / & / \\
\hline linezolid & 1 & / & 2 & 2 & / & / & / & / \\
\hline polymyxin $\mathrm{E}$ & / & / & / & / & 1 & / & / & 1 \\
\hline tigecycline & 2 & $/$ & 1 & / & 2 & / & 1 & / \\
\hline daptomycin & 2 & / & 4 & 1 & I & I & I & / \\
\hline
\end{tabular}

Table 2 Antibacterial activity of RD22 and RD53 against a panel of Gram-positive and Gram-negative pathogens

\begin{tabular}{|c|c|c|c|c|}
\hline & \multicolumn{2}{|l|}{ Organism } & \multicolumn{2}{|l|}{ MICa } \\
\hline & & & RD22 & RD53 \\
\hline \multirow[t]{17}{*}{ Gram+ } & S. aureus & ATCC25923(MSSA $\left.{ }^{b}\right)$ & 1 & 1 \\
\hline & & CMCC26003(MSSA $\left.{ }^{b}\right)$ & 1 & 0.5 \\
\hline & & ATCC43300 (MRSA $\left.{ }^{b}\right)$ & 1 & 1 \\
\hline & & 10 Clinical isolates (MRSA ${ }^{b}$ ) & 0.25 & $0.25-2$ \\
\hline & E. faecalis & ATCC29212(VSEd) & 2 & 2 \\
\hline & & ATCC51299(VREd) & 2 & 2 \\
\hline & & 5 Clinical isolates (VSEd) & 1 & 1 \\
\hline & E. faecium & ATCC35667 & 1 & 2 \\
\hline & & 5 Clinical isolates (VREd) & 1 & 1 \\
\hline & & 3 Clinical isolates (VSE ${ }^{d}$ ) & 1 & $0.5-1$ \\
\hline & S. epidermidis & CMCC26069(MSSEC) & 1 & 1 \\
\hline & & 2 Clinical isolates(MSSEC) & 1 & 1 \\
\hline & & 1 Clinical isolates (MRSEc) & 1 & 1 \\
\hline & S. pyogenes & СMCС32006 & 1 & 1 \\
\hline & & 3 Clinical isolates & 0.5 & $2-4$ \\
\hline & H. pylori & Sydney Strain 1 & n.a. & 0.5 \\
\hline & S. pneumoniae & 3 Clinical isolates (PRSP)e & $0.5-1$ & $2-4$ \\
\hline \multirow[t]{10}{*}{ Gram- } & A. baumannii & ATCC19606 & 4 & 4 \\
\hline & P. aeruginosa & ATCC27853 & 16 & 16 \\
\hline & E. coli & ATCC25922 & 16 & 8 \\
\hline & & 9 Clinical isolates $(E S B L+)^{f}$ & $2-4$ & $2-4$ \\
\hline & & 4 Clinical isolates (ESBL-) ${ }^{f}$ & 4 & $4-8$ \\
\hline & K. pneumoniae & ATCC13883 & 16 & 16 \\
\hline & & 3 Clinical isolates $(E S B L+)^{f}$ & 4 & $4-8$ \\
\hline & & 4 Clinical isolates(ESBL-) ${ }^{f}$ & $4-8$ & \\
\hline & & 4 Clinical isolates(CRE)g & $4-8$ & $8-16$ \\
\hline & S. flexneri & ATCC51081 & 2 & 1 \\
\hline
\end{tabular}

aMIC's are given in unit of $\mu \mathrm{g} \mathrm{mL}-1$

bMSSA (or MRSA): methicillin-sensitive (or resistant) Staphylococcus aureus

CMSSE (or MRSE): methicillin-sensitive (or resistant) Staphylococcus epidermidis

dVSE (or VRE): vancomycin sensitive (or resistant) Enterococcus

ePRSP: penicillin-resistant Staphylococcus pneumonia

ESBL: extended spectrum beta-lactamases

gCRE: carbapenem-resistant Enterobacteriaceae

also effective toward a broad range of clinically isolated, difficultto-treat, multidrug-resistant bacteria, including S. aureus (MRSA, 10 strains), E. faecalis (VSE, 5 strains), E. faecium (VRE, 5 strains; VSE, 3 strains), S. epidermidis (MSSE, 2 strains; MRSE, 1 strain), S. pyogenes (3 strains), S. pneumonia (3 strains), E. coli (ESBL+, 9 strains; ESBL-, 4 strains), K. pneumonia (ESBL+, 3 strains, ESBL-, 4 strains, and CRE, 4 strains). Compared to RD53, RD22 displayed equally potent antimicrobial activity against all the aforementioned standard reference Gram-positive and Gramnegative type bacteria and clinical isolates as well.

The clinical significance of the first-line bacteriostatic antibiotics, e.g., vancomycin, linezolid, et al., warrants no further elaboration. However, in some serious clinical situations, such as endocarditis, meningitis, osteomyelitis, neutropenia, bactericidal action is necessary because of the reduced rates of metabolism and cell division, the poor immunologic competence or the poor drug penetration ${ }^{53}$. The bactericidal activity of RD22 and RD53 were compared to multiple first-line antibiotics using the time-kill assay (Fig. 4). Methicillin-resistant S. aureus, Vancomycinresistant E. faecalis, and polymyxin E-resistant A. baumannii were chosen as the tested Gram-positive and Gram-negative strains, respectively. MRSA (ATCC43300) were grown to early exponential phase and challenged with $10 \times$ MIC of vancomycin, linezolid, tigecycline, respectively (Fig. 4a). In the first $4 \mathrm{~h}$, bacteria concentration remained steady in the presence of vancomycin and linezolid and then gradually dropped by twothree orders of magnitude within $24 \mathrm{~h}$. Tigecycline exhibited a higher degree of bactericidal activity than vancomycin and linezolid. In comparison, RD22 (Fig. 4b) and RD53 (Fig. 4c) were more potent bactericidal agents than vancomycin, linezolid or tigecycline. Bacteria challenged by as low as $2.5 \times$ MIC lowered the bacteria concentration by four order of magnitude of bacteria within as short as $2 \mathrm{~h}$. RD22 and RD53 also exhibits bactericidal activities to VRE (ATCC51299) (Fig. 4e, f). They lowered the concentration of VRE by over 4 orders of magnitude within $2 \mathrm{~h}$ by $(5-10) \times$ MIC. In comparison, linezolid, tigecycline did not exhibit strong bactericidal activity and bacterial concentration dropped by less than 2 orders of magnitude within $24 \mathrm{~h}$. Daptomycin was able to reduce the cell concentration by over 4 orders of magnitude within $8 \mathrm{~h}$ (Fig. 4d). Polymyxin E-resistant A. baumannii (MIC $=256 \mu \mathrm{g} \mathrm{mL}-1$, stored in the lab) was obtained by serial passages of cells at sub-MIC concentration of polymyxin E during resistance acquisition of pathogens. RD53 at $10 \times$ MIC was able to reduce the cell concentration by over 4 orders of magnitude within $6 \mathrm{~h}$ (Fig. 4i) while at the same dose of tigecycline and RD22 reduced the cell concentration by around 3 orders of magnitude (Fig. 4g, h). And RD53 at $8 \times$ MIC could also reduce the cell concentration of $H$. pylori by more than 4 orders of magnitude within $2 \mathrm{~h}$ (Supplementary Figure 132). So, the time-kill assay proved that $\mathbf{R D 2 2}$ and $\mathbf{R D 5 3}$ are potent bactericidal agents. They can potentially complement bacteriostatic antibiotics in serious bacterial infections to hosts, whose immune response is compromised.

Resistance studies of RD22 and RD53. Ease of resistance acquisition toward RD22 and RD53 was evaluated using MRSA, VRE and A. baumannii as tested bacteria (Fig. 5). Serial passages of MRSA in the presence of sub-MIC levels of levofloxacin over a 

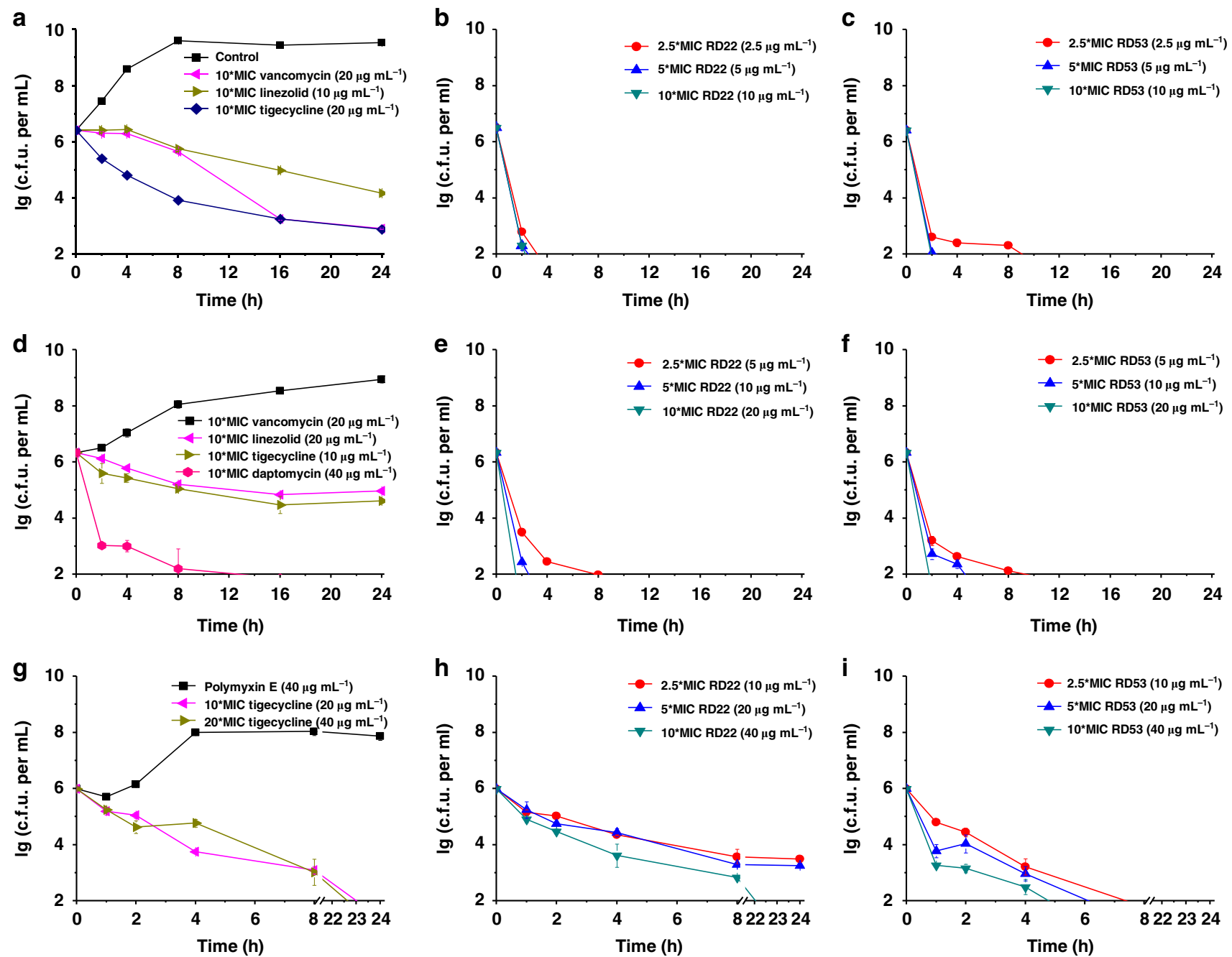

Fig. 4 Time-dependent killing of pathogens by RD22 or RD53 compared to clinical antibiotics. MRSA (ATCC43300) challenged with a vancomycin, linezolid, tigecycline; b RD22; c RD53. VRE (ATCC51299) challenged with d vancomycin, linezolid, tigecycline, daptomycin; e RD22; f RD53. Polymyxin Eresistant Acinetobacter baumannii. challenged with $\mathbf{g}$ polymyxin E, tigecycline $\mathbf{h} \mathbf{R D 2 2}$; $\mathbf{\text { RD53}}$. Pathogens were grown to early exponential phase and challenged with $\mathbf{R D 2 2 / 5 3}$ or clinical antibiotics. Data are representative of three independent experiments. Error bars indicate s.d.
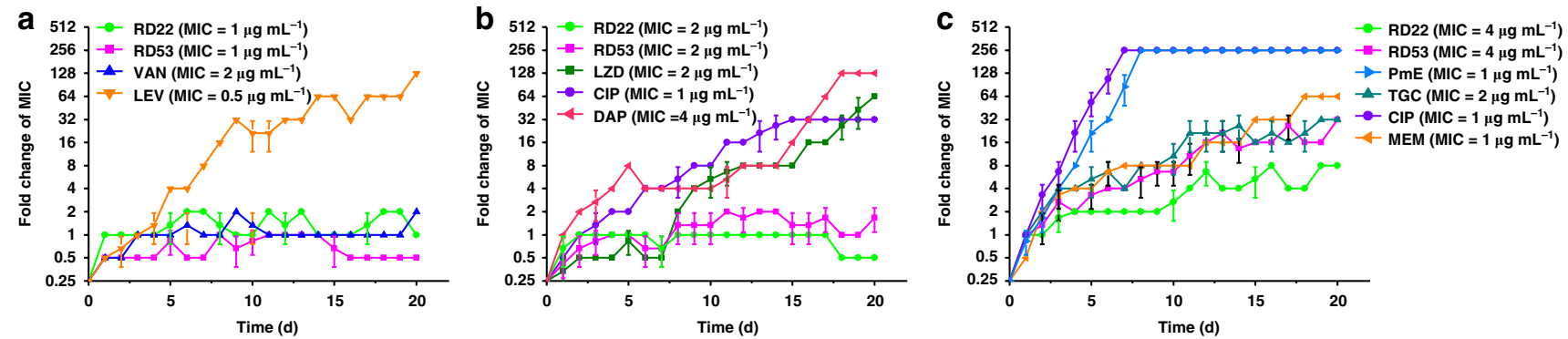

Fig. 5 Resistance acquisition of pathogens during serial passaging in sub-MIC levels of antimicrobials. The y axis is the fold change of MIC. a MRSA (ATCC43300) towards RD22 ( $\mathrm{MIC}=1 \mu \mathrm{g} \mathrm{mL}-1$, tested up to $2 \times \mathrm{MIC})$, RD53 (MIC $=1 \mu \mathrm{g} \mathrm{mL}-1$, tested up to $1 \times \mathrm{MIC}$ ), levofloxacin (MIC $=0.5 \mu \mathrm{gL}{ }^{-1}$, tested up to $128 \times \mathrm{MIC})$, and vancomycin (MIC $=2 \mu \mathrm{g} \mathrm{mL}-1$, tested up to $2 \times \mathrm{MIC}) . \mathbf{b}$ VRE (ATCC51299) toward $\mathbf{R D 2 2}(\mathrm{MIC}=2 \mu \mathrm{g} \mathrm{mL}-1$, tested up to $1 \times$ MIC), RD53 (MIC $=2 \mu \mathrm{g} \mathrm{mL}-1$, tested up to $2 \times M I C)$, linezolid (LZD, MIC $=2 \mu \mathrm{g} \mathrm{mL}-1$, tested up to $64 \times M I C)$, ciprofloxacin $\left(C I P, M I C=1 \mu g L^{-1}\right.$, tested up to $32 \times \mathrm{MIC})$, and daptomycin (DAP, MIC $=4 \mu \mathrm{g} \mathrm{mL}-1$, tested up to $128 \times \mathrm{MIC})$. c A. baumannii (ATCC19606) toward RD22 $(\mathrm{MIC}=4 \mu \mathrm{mL}-1$, tested up to $8 \times \mathrm{MIC}), \mathbf{R D 5 3}(\mathrm{MIC}=4 \mu \mathrm{g} \mathrm{mL}-1$, tested up to $32 \times \mathrm{MIC})$, polymyxin $\mathrm{E}\left(\mathrm{PmE}, \mathrm{MIC}=1 \mu \mathrm{g} \mathrm{mL}{ }^{-1}\right.$, tested up to $\left.256 \times \mathrm{MIC}\right)$, tigecycline (TGC, $\mathrm{MIC}=2 \mu \mathrm{g} \mathrm{mL}-1$, tested up to $32 \times \mathrm{MIC})$, and ciprofloxacin $\left(\mathrm{CIP}, \mathrm{MIC}=1 \mu \mathrm{g} \mathrm{mL} \mathrm{L}^{-1}\right.$, tested up to $\left.256 \times \mathrm{MIC}\right)$, meropenem $(\mathrm{MEM}, \mathrm{MIC}=1 \mu \mathrm{g} \mathrm{mL}-1$, tested up to $64 \times$ MIC). Data are representative of three independent experiments. Error bars indicate s.d. 
period of 20 days raised the MIC by 128 folds, i.e. rendering the strain highly resistant to levofloxacin. In comparison, MRSA did not develop resistance toward vancomycin, exactly the reason why vancomycin serves as the last-line antibiotic against MRSA. It is very interesting to note that MRSA also did not acquire a resistance by genomic mutations toward RD22 and RD53 as well (Fig. 5a). In a separate experiment, VRE developed resistance toward linezolid, ciprofloxacin and daptomycin by serial passages in 20 days, with a raise of MIC's by 32-128 folds while no obvious resistance was observed with RD22 and RD53 by VRE (Fig. 5b). The Gram-negative A. baumannii readily acquired resistance by genomic mutations toward polymyxin $\mathrm{E}$ and ciprofloxacin with a 256 enhancement of MIC in as short as 7 days. Bacteria did develop resistance toward meropenem, tigecycline, RD22 and RD53 as well, but to a much reduced degree, i.e. 64-, 32-, 8-, and 32 -fold enhancement of MIC within 20 days, respectively (Fig. 5c). It has become evident that either Gram-positive or Gram-negative pathogens were found not to readily acquire resistance by genomic mutations toward RD22 and RD53. Analogously, E. coli, E. faecalis and S. aureus were found not to acquire resistance toward RD53 (Supplementary Figure 133). Development of resistance toward an antibiotic by bacteria is an evolutionary inevitability and thereby imposes a limit on the lifespan of any given antibiotic. And resistance was involved in clinical failure of some antibiotic administration regimes. This is especially evident because the antibiotic levels at some points during treatment are in the sub-inhibitory range $\mathrm{e}^{54}$. Therefore, the low tendency of resistance acquisition toward RD22 and RD53 by various pathogens greatly enhances their potentials for further drug development.

Probing mechanism of action. The mechanism of action was investigated, with RD53 as an example. First, the lack of noticeable morphological variation of MRSA and A. baumannii, as indicated by the SEM images (Fig. 6) with and without RD53, suggested that RD53 did not destruct either the cell wall or membrane. This observation was further corroborated by the fact that RD53 exhibits low haemolytic activity with a Lysis 20 value of above $100 \mu \mathrm{g} \mathrm{mL} \mathrm{m}^{-1}$. Second, a resistant strain of A. baumannii to RD53 (RD53-Ab-20th) was produced by serial passages of ATCC19606 exposed to sub-MIC concentration of RD53 over a period of 20 days, with the MIC of RD53 raised from $4 \mu \mathrm{g} \mathrm{mL}-1$ to $128 \mu \mathrm{g} \mathrm{mL}-1$. Yet, the phenotypic profile of RD53-Ab-20th with respect to susceptibility to other antibacterial drugs, e.g., tigecycline (TGC), ciprofloxacin (CIP), polymyxin E (PmE), levofloxacin (LEV), and ampicillin-sulbactam (SAM), remains unchanged compared to ATCC19606, suggesting the lack of cross-resistance between RD53 and these antibiotics (Supplementary Table 9). Third, selective pressure on antibacterial targets could also be measured by genomic profile of mutational resistant bacteria. So, the genomic analysis was carried out for clues on the potential mechanism of action of RD53. Comparative analysis revealed the existence of eight nonsynonymous single nucleotide variants (SNV) and one nonframeshift deletion (Table 3), which belong to resistance/tolerance-associated genes encoding efflux pumps, metabolism, DNA replication or transcriptional regulation related proteins ${ }^{55,56}$. It is interesting to find the mutation in rsmJ which encodes 16S rRNA (guanine1516 - N2) - methyltransferase. Notably, the mutation in $16 \mathrm{~S}$ rRNA methyltransferase has been shown to cause kasugamycin resistance in E. coli ${ }^{57,58}$. These clues together have formulated a solid framework for further investigation of the mechanism of action of RD53.

Structure-activity relationship investigation. The structureactivity relationship of these rhodamine-type antibiotic
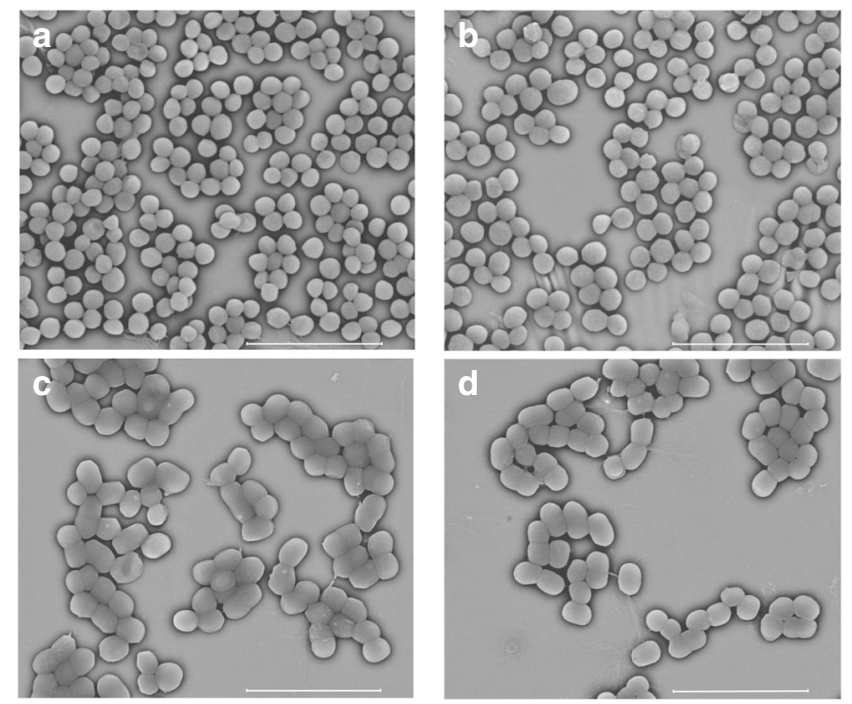

Fig. 6 SEM images for MRSA and A. baumannii with or without treatment of RD53. a MRSA without treatment of RD53. b MRSA after incubation with $8 \times$ MIC ( $8 \mu \mathrm{g} \mathrm{mL}^{-1}$ ) RD53 for 4 h. c A. baumannii without treatment of RD53. d $A$. baumannii after incubation with $8 \times \mathrm{MIC}\left(32 \mu \mathrm{g} \mathrm{mL}{ }^{-1}\right)$ RD53 for 4 h. SEM images were taken by FEI Nova NanoSEM 230. The SEM was operated in the high vacuum at an accelerating voltage of $7.5 \mathrm{kV}$. Scale bars represent $5 \mu \mathrm{m}$

compounds was examined. Out of the 70 members of this rhodamine library, 37 compounds exhibit antibiotic activity of varying potency (Fig. 3). This fact alone strongly suggested that their common structural motif is the pharmacologically active motif, which is the rhodamine core. To verify this hypothesis, we have prepared a few closely related analogs (C1-2, and RD71-74, Fig. 7). First, $\mathbf{C} 1$ and $\mathbf{C} 2$ differ from the RD53 in that $\mathbf{C} 1$ misses the oxygen atom of the rhodamine core compared to RD53, and $\mathbf{C} 2$ has one more aniline moiety removed compared to $\mathbf{C 1}$. Both C1 and C2 exhibited no appreciable antibiotic activity with their MIC's higher than $64 \mu \mathrm{g} \mathrm{mL}-1$ toward both ATCC43300 and ATCC19606 (Fig. 7a). Second, the peripheral group of the rhodamine core or the adamantine moiety could be modified without inhibiting the antibiotic activity as long as the rhodamine core remains intact, as showcased with RD71-74 (Fig. 7b, c). The aforementioned hypothesis was further supported by the following experimental results that none of the starting materials (S1-S69) for preparations of these rhodamine analogs (RD1RD69) display antibiotic activity (MIC $>64 \mu \mathrm{g} \mathrm{mL}^{-1}$ ) (Supplementary Table 3 ). This reiterates the necessity of the rhodamine core for antibiotic activity. Additional support to this hypothesis comes from the ten compounds listed in Fig. 7d. These compounds predominantly adopt the ring-closed form and therefore they do not actually have the rhodamine motif. And indeed, none of them have been found to exhibit antibiotic activity during the phenotypic screening. The discovery of the above structureactivity relationship is important as it provides a general guideline for further structural modification, if warranted in the future drug-development endeavors.

\section{Discussion}

Diversity-oriented synthesis of xanthene library attracted attention for discovery of original bioactive compounds through phenotypic screening. This has promoted search for a mild xanthene synthesis allowing facile installation of functional, topographical and stereochemical diversity. We have devised a mild, one-step, high-yielding and readily scalable xanthene synthesis, by reacting a rationally constructed dilithium species 
Table 3 Mutations present in RD53-resistant A. baumannii

\begin{tabular}{|c|c|c|c|}
\hline Mutation $^{a}$ & Gene name ${ }^{b}$ & Definition & Amino acid change \\
\hline \multicolumn{4}{|l|}{ SNPS } \\
\hline $\mathrm{G} \rightarrow \mathrm{C}$ & kup & KUP system potassium uptake protein & A373P \\
\hline$A \rightarrow T$ & $\operatorname{mex} B$ & multidrug efflux pump & S617T \\
\hline$A \rightarrow G$ & $\operatorname{mex} B$ & multidrug efflux pump & L149P \\
\hline $\mathrm{G} \rightarrow \mathrm{T}$ & $\operatorname{trkH}, \operatorname{trkG}, k \operatorname{tr} B$ & trk system potassium uptake protein & S14R \\
\hline $\mathrm{G} \rightarrow \mathrm{A}$ & $n p d A$ & NAD-dependent deacetylase & A14T \\
\hline $\mathrm{C} \rightarrow \mathrm{T}$ & rsmJ & 16S rRNA (guanine1516-N2)-methyltransferase & A228T \\
\hline $\mathrm{C} \rightarrow \mathrm{T}$ & hisD & histidinol dehydrogenase & V257A \\
\hline $\mathrm{G} \rightarrow \mathrm{A}$ & etk-wzc & tyrosine-protein kinase Etk/Wzc & D569N \\
\hline \multicolumn{4}{|l|}{ InDel } \\
\hline GAACCT $\rightarrow-$ & DPO3G & DNA polymerase III subunit gamma/tau & 441_442del \\
\hline
\end{tabular}

with alkyl/aryl/alkenyl substituted esters, anhydrides, lactones and even some lactams and synthesized a focused library of xanthene dyes with 70 members. Through in vitro screening against various multi-drug-resistant pathogens, we found that it is a rich source of hit compounds exhibiting potent bactericidal activity. Particularly, RD22 and RD53 are two antibacterial hits exhibiting favorable properties for further drug development. First, the growth of a wide-spectrum of Gram-positive and Gramnegative pathogens was inhibited by RD22 and RD53 with a low MIC. They are a potent bactericidal agent and a low dose at $(2.5-10) \times$ MIC could reduce the concentration of methicillinresistant $S$. aureus, vancomycin-resistant E. faecalis, polymyxin $\mathrm{E}$ resistant $A$. baumannii by 4 orders of magnitude within ca. $4 \mathrm{~h}$, more quickly than the current first-line and last-resort antibiotics, e.g. vancomycin, linezolid, tigecycline, and daptomycin. While the pathogens readily acquire resistance toward these existing antibiotics, exposing the cells toward sub-MIC concentration of RD22 and RD53 did not readily induce emergence of resistance. Fourth, they have a large therapeutic index of over 100, which is defined by the Lysis20/MIC. Our work is reminiscent of the discovery of Prontosil, the first antibiotic, from an azo dye library by Gerhard Domagk. The successful treatment of streptococcus infection of her daughter foreran what was later known as the Golden Age of Antibiotics. With this manuscript, we want to share with the community that dye libraries of high structural diversity could potentially be that sought-after chemical space of alternative antibiotics when no other natural or unnatural chemical space are currently offering abundant hit compounds for further drug development.

\section{Methods}

Construction and characterization of the rhodamine library. Details of synthesis and characterization of RD1-RD74, C1-C2 employed in this manuscript were included in the Supplementary Methods section (Supplementary Figure 383393). Structures of substrates (S1-S69) can be found in Supplementary Figure 1. General procedures B was illustrated as the general synthetic route for the preparation of rhodamine RD1-72 via nucleophilic condensation of a dilithium reagent $\mathbf{1}$ with various substrates S1-S70. The UV-Vis absorption and fluorescence emission spectra in $\mathrm{pH}=7.4$ PBS with $1 \%$ DMSO was shown in Supplementary Figures 2-69, and spectral properties were summarized in Supplementary Table 1. For ${ }^{1} \mathrm{H}-/{ }^{13} \mathrm{C}-\mathrm{NMR}$ and MS spectra see Supplementary Figures 135-382.

Bacterial cell culturing. The following bacterial strains were used: Staphylococcus aureus ATCC25923 (American type culture collection, Beijing Zhongyuan Ltd., cat. no. 25923), CMCC26003 (National Center for Medical Culture Collections, cat. no. 26003), ATCC43300 (American type culture collection, Beijing Zhongyuan Ltd., cat. no. 43300), Enterococcus faecalis ATCC29212 (American type culture collec tion, Beijing Zhongyuan Ltd., cat. no. 29212), ATCC51299 (American type culture collection, Beijing Zhongyuan Ltd., cat. no. 51299), Enterococcus faecium ATCC35667 (American type culture collection, Beijing Zhongyuan Ltd., cat. no. 35667), Staphylococcus epidermidis CMCC26069 (National Center for Medical
Culture Collections, cat. no. 26069), Streptococcus pyogenes CMCC32006 (National Center for Medical Culture Collections, cat. no. 32006), Helicobacter pylori Sydney Strain 1 (BioVector NTCC Inc., cat. no. HpSS1), Acinetobacter baumannii ATCC19606 (American type culture collection, Beijing Zhongyuan Ltd., cat. no. 19606), Pseudomonas aeruginosa ATCC27853 (American type culture collection, Beijing Zhongyuan Ltd., cat. no. 27853), Escherichia coli ATCC25922 (American type culture collection, Beijing Zhongyuan Ltd., cat. no. 25922), Shigella flexneri ATCC51081 (American type culture collection, Beijing Zhongyuan Ltd., cat. no. 51081). The clinical strain isolated from patients was provided and tested by Shanghai Huashan Hospital. Bacteria were cultured in nutrient media, either cation-adjusted Mueller-Hinton broth (S.aureus, E.faecalis, E.faecium, S.epidermidis, S.pyogenes, A.baumannii, P.aeruginosa, E.coli, S.flexneri) at $37^{\circ} \mathrm{C}$ in shaking flasks or Brucella agar supplemented with $5 \%$ defibrinated sheep blood (H.pylori) under microaerophile conditions at $37^{\circ} \mathrm{C}$. The abbreviations and strains of all the organisms used in this article are summarized in Supplementary Table 4.

Minimum inhibitory concentration (MIC). MIC was determined by broth microdilution for most species and by agar dilution method for Helicobacter pylori according to CLSI guidelines. Cell concentration was adjusted to approximately $5 \times 10^{5}$ cells per $\mathrm{mL}$. For broth microdilution, the test medium was cation-adjusted Mueller-Hinton broth (MHB). After $20 \mathrm{~h}$ of incubation at $37^{\circ} \mathrm{C}$, the MIC was defined as the lowest concentration of antibiotic with no visible growth. As for agar dilution method, the test medium was supplemented Brucella agar plates. The plates were incubated under microaerophile conditions for $48 \mathrm{~h}$ at $37^{\circ} \mathrm{C}$. The MIC was defined as the lowest concentration that completely inhibited visible growth as compared to the drug-free control. Expanded antibacterial spectrum to clinical isolates was tested at Shanghai Huashan Hospital. Experiments were performed with biological replicates. MIC's of reference antibiotics against different organisms are summarized in Supplementary Table 5.

Time-kill curves. An overnight culture of cells (methicillin resistant $S$. aureus ATCC43300; vancomycin resistant Enterococcus ATCC51299; polymyxin Eresistant A. baumannii which was induced from ATCC19606) was diluted 1:10,000 in $\mathrm{MHB}$ and incubated at $37^{\circ} \mathrm{C}$ with aeration at 220 r.p.m. for $2 \mathrm{~h}$ (early exponential). Bacteria were then challenged with RD22 or RD53 at $2.5 \times$ MIC, $5 \times$ MIC, $10 \times$ MIC (a desirable concentration at the site of infection) comparing with antibiotics clinically used in culture tubes at $37^{\circ} \mathrm{C}$ and 220 r.p.m. At intervals, $100 \mu \mathrm{l}$ aliquots were removed, tenfold serially diluted suspensions were plated on MHA plates and incubated at $37^{\circ} \mathrm{C}$ overnight. H. pylori SS1 cultured overnight $\left(\right.$ c. $2 \times 10^{8}$ c.f.u. per $\mathrm{mL}$ ) of Brucella broth supplemented with $2 \%$ heat-inactivated fetal bovine serum (FBS broth) was diluted 1:100 with the same medium containing $1 \times$ MIC, $2 \times$ MIC, $4 \times$ MIC, $8 \times$ MIC RD22 or RD53 comparing with antibiotics clinically used at $37^{\circ} \mathrm{C}$, under microaerophile conditions and 220 r.p.m. At intervals, $100 \mu \mathrm{L}$ aliquots were removed, tenfold serially diluted suspensions were plated on supplemented Brucella agar plates and incubated at $37^{\circ} \mathrm{C}$ for $48 \mathrm{~h}$. Colonies were counted and c.f.u. per $\mathrm{mL}$ was calculated. Experiments were performed with biological replicates.

Resistance study. An overnight culture of cells (methicillin resistant $S$. aureus ATCC43300; vancomycin resistant Enterococcus ATCC51299; A. baumannii ATCC19606 were grown in the presence of subinhibitory levels $(0.25 \times$ MIC) of RD22 or RD53 in order to increase the probability of obtaining resistant mutants. Antibiotics clinically used were included as a control. Next, the cells were added to RD22 or RD53 present at $0.25 \times$ MIC, $0.5 \times$ MIC, $1 \times$ MIC, $2 \times$ MIC and $4 \times$ MIC. At $24 \mathrm{~h}$ intervals, the cultures were checked for growth. Cultures from the highest concentrations $\left(\mathrm{C}_{\mathrm{n}}, \mathrm{n}=\right.$ passage number $)$ that allowed growth $\left(\mathrm{OD}_{600} \geq 2\right)$ were diluted 1:100 into fresh media containing $0.25 \times C_{n}, 0.5 \times C_{n}, 1 \times C_{n}, 2 \times C_{n}$ and 

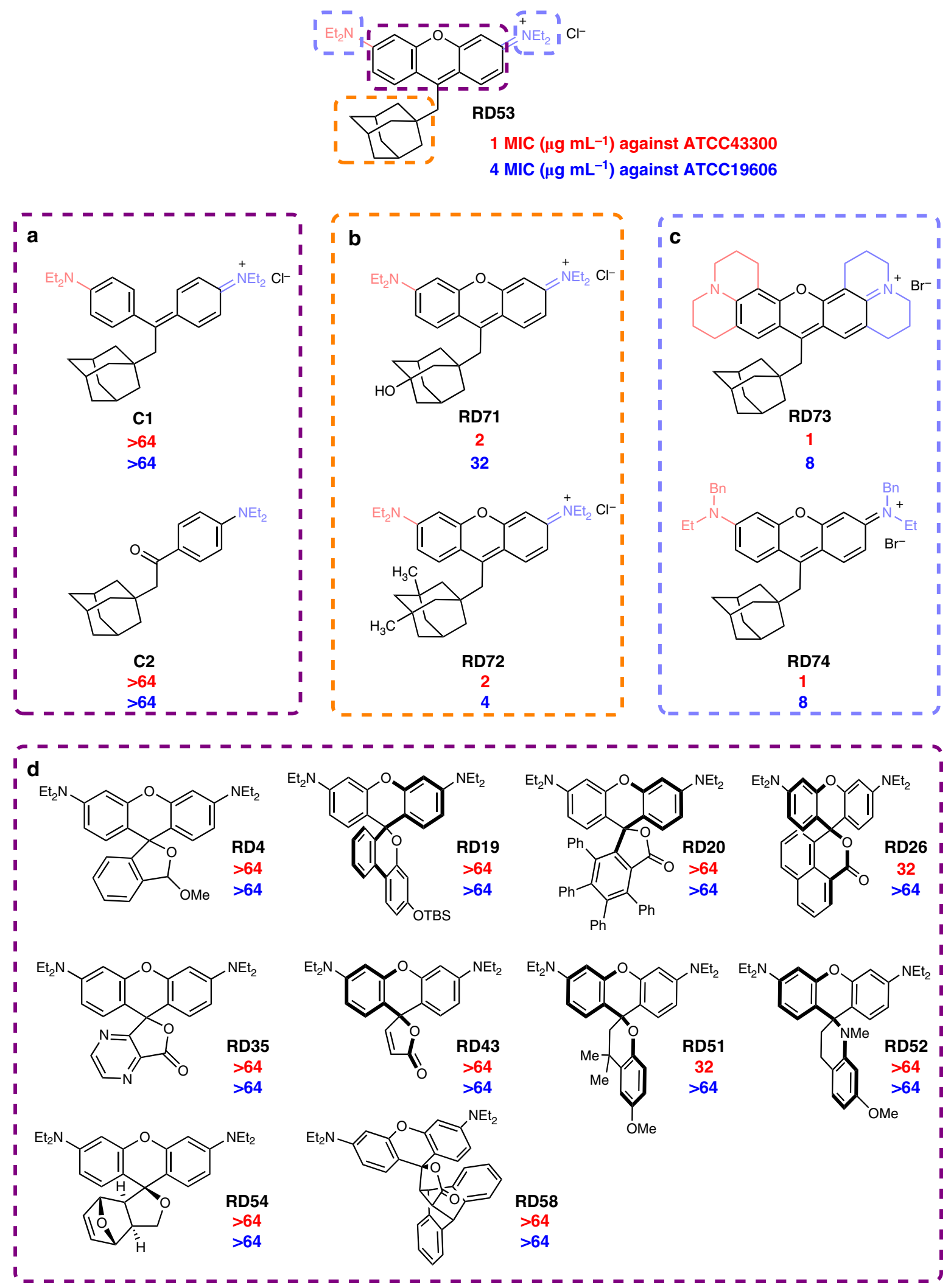

Fig. 7 Structure-activity relationship investigation of the rhodamine library. a RD53 analogs without the rhodamine core; b RD53 analogs with the adamantine moiety modified; c RD53 analogs with the diethylamino group modified. d Rhodamine dyes mainly adopted the ring-close forms exhibit no antibacterial activity against ATCC43300 or ATCC19606, with MIC $>64 \mu \mathrm{g} \mathrm{mL}-1$ 
$4 \times C_{n}$ of RD22 or RD53. This serial passaging was repeated daily for 20 days. Experiments were performed with biological replicates. Results of absolute fold change of MIC of 30 colonies purified from the 5th and 10th passage were shown in Supplementary Table 7. Results of spontaneous frequency of resistance study were shown in Supplementary Table 8.

\section{Scanning electron microscope (SEM) analysis for MRSA and A. baumannii.} The cells were grown in the MHB broth medium to around $10^{7}$ c.f.u. per $\mathrm{mL}$ and collected before drug treatment and $4 \mathrm{~h}$ after treatment of $8 \times$ MIC RD53 $(8 \mu \mathrm{g} \mathrm{mL}-1$ for MRSA and $32 \mu \mathrm{g} \mathrm{mL} L^{-1}$ for A. baumannii). The cells were fixed in $2.5 \% \mathrm{glu}-$ taraldehyde (prepared in PBS buffer) for $2 \mathrm{~h}$, and washed with PBS buffer twice after fixation. Then the cells were dehydrated in a series of ethanol solutions which are $30 \%, 40 \%, 50 \%, 60 \%, 70 \%, 80 \%, 90 \%, 100 \%$ ethanol, sequentially for $15 \mathrm{~min}$ each, and finally suspended in pure tert-butanol for $15 \mathrm{~min}$. The cells were criticalpoint dried and coated with gold following by scanning electron microscope analysis (FEI Nova NanoSEM 230). The SEM was operated in the high vacuum at an accelerating voltage of $7.5 \mathrm{kV}$.

Haemolytic activity: red blood cell (RBC) lysis assay (Lysis20). Lysis20 values of RD22 and RD53 were measured as the concentration of compound that lyses $20 \%$ or less of sheep red blood cells (RBC). The therapeutic index (Lysis20/MIC) was over 100 when comparing the MIC against MRSA to the observed Lysis20. RBC lysis assays were performed on mechanically defibrinated sheep blood. $1.5 \mathrm{~mL}$ of blood was placed into a microcentrifuge tube and centrifuged at $8609 \times g$ for $10 \mathrm{~min}$. The supernatant was removed and the cells were resuspended with $1 \mathrm{~mL}$ of $5 \%$ glucose solution. The suspension was centrifuged as previously, the supernatant was removed, and cells were resuspended two more times. The final cell suspension was diluted twentyfold with $5 \%$ glucose solution. The twentyfold suspension dilution was then aliquoted into microcentrifuge tubes containing compound serially diluted in $5 \%$ glucose solution. Triton X ( $1 \%$ by volume) served as a positive control ( $100 \%$ lysis marker) and sterile $5 \%$ glucose solution served as a negative control ( $0 \%$ lysis marker). Samples were then placed in an incubator at $37^{\circ} \mathrm{C}$ and shaken at 200 r.p.m. After $1 \mathrm{~h}$, the samples were centrifuged at $8609 \times g$ for $10 \mathrm{~min}$. The absorbance of the supernatant was measured with a UV spectrometer at a $330 \mathrm{~nm}$ wavelength.

Mammalian cytotoxicity of RD53. Cytotoxicity was determined on normal human kidney 2 cells HK-2 cells human renal proximal tubular cells (Cell Bank of Chinese Academy of Science, cat. no. SCSP-511), HUVEC Primary Umbilical Vein Endothelial Cells (American type culture collection, cat. no. PCS-100-013) and HeLa human cervical carcinoma cells (Cell Bank of Chinese Academy of Science, cat. no. TCHu 187). Briefly, HK-2 and HeLa cells were cultured in RPMI1640 medium supplemented with FBS, and HUVEC were cultured in DMEM, high glucose medium supplemented with FBS. When cells reached $\sim 80 \%$ confluence, they were harvested and resuspended in the growth medium to $\sim 2.0 \times 10^{5}$ cells $\mathrm{mL}^{-1} .100 \mu \mathrm{L}$ cells were inoculated into 96 well plate and incubated at $37^{\circ} \mathrm{C}$ with $5 \% \mathrm{CO}_{2}$ for $4.5 \mathrm{~h}$. Then, $0.25 \mu \mathrm{L}$ of twofold serial compound dilutions in DMSO to $99.75 \mu \mathrm{L}$ of media were added to the plate and incubated at $37^{\circ} \mathrm{C}$ with $5 \% \mathrm{CO}_{2}$ for $43 \mathrm{~h}$. For cell viability test, $20 \mu \mathrm{L} 0.5 \%$ MTT solution were added to the plate and the supernatant was discarded after $4 \mathrm{~h}$ incubation. Then $150 \mu \mathrm{L}$ DMSO were added to dissolve formazan and the A490nm (OD490) was measured. $50 \%$ cell cytotoxicity $\left(\mathrm{CC}_{50}\right)$ was analyzed in GraphPad Prism. Results are shown in Supplementary Figure 134.

Whole-genome sequencing. A single colony of various bacteria was grown until the $\mathrm{OD}_{600}$ value reaches 0.3 . The Genomic DNA was extracted and subjected to paired-ends Illumina sequencing. The whole genome sequencing of RD53-Ab-20th and ATCC19606 was acquired on an Illumina HiSeq 2000 system (Illumina Inc; San Diego, USA). The manufacturer's protocol (NEBNext ${ }^{\circledR}$ Ultra $^{\mathrm{m}}$ DNA Library Prep Kit for Illumina ${ }^{\circledR}$ ) was followed to construct the Next Generation Sequencing Library Preparations. The extracted genomic DNA $(1 \mu \mathrm{g})$ was sonicated to random fragments containing $500 \mathrm{bp}$ or less. The End Prep Enzyme Mix was employed to treat the resulting fragments for end-repairing, $5^{\prime}$-phosphorylation and dA-tailing in one step. T-A ligation was carried out to add adaptors to both ends. Adaptorligated DNA was then sized-selected with AxyPrep Mag PCR Clean-up to recover the fragments of ca. $410 \mathrm{bp}$, the approximate insert size of which is $350 \mathrm{bp}$. The resulting samples were subsequently amplified by PCR for 8 cycles. The PCR products were treated with the AxyPrep Mag PCR Clean-up kit, validated with Agilent 2100 Bioanalyzer, and quantified by Qubit2.0 Fluorometer. Then libraries with different indices were multiplexed and loaded onto an Illumina HiSeq instrument. Sequencing was performed using a 2x150 paired-end (PE) configuration. The image analysis and base calling were carried out with the HiSeq Control Software (HCS) + OLB + GAPipeline-1.6 (Illumina) on the HiSeq instrument. The sequences of adaptors, PCR primers, content of $\mathrm{N}$ bases more than $10 \%$ and bases with a quality lower than 20 were removed using Cutadapt (V1.9.1). The clean data was mapped to the reference genome with BWA (V0.7.12) and duplicate-removed by Picard (V1.119). The Unified Genotyper calls single-nucleotide variant (SNV), insertions/deletions (InDel) with the GATK (V3.4.6) software. Annotation for
SNV/InDel was performed with Annovar (V21 Feb 2013). Pindel and CNVnator were employed to analyze genomic structure variation.

\section{Data availability}

The data that support the findings of this study are available from the corresponding authors on request.

Received: 28 January 2018 Accepted: 21 December 2018 Published online: 16 January 2019

\section{References}

1. Levy, S. B. \& Marshall, B. Antibacterial resistance worldwide: causes, challenges and responses. Nat. Med. 10, S122-S129 (2004).

2. Davies, J. \& Davies, D. Origins and evolution of antibiotic resistance. Microbiol. Mol. Biol. Rev. 74, 417-433 (2010).

3. Blair, J. M., Webber, M. A., Baylay, A. J., Ogbolu, D. O. \& Piddock, L. J. V. Molecular mechanisms of antibiotic resistance. Nat. Rev. Microbiol. 13, 42-51 (2015)

4. Brown, E. D. \& Wright, G. D. Antibacterial drug discovery in the resistance era. Nature 529, 336-343 (2016).

5. Alanis, A. J. Resistance to antibiotics: are we in the post-antibiotic era? Arch Med. Res. 36, 697-705 (2005).

6. Wang, J. et al. Platensimycin is a selective FabF inhibitor with potent antibiotic properties. Nature 441, 358-361 (2006).

7. Lewis, K. Platforms for antibiotic discovery. Nat. Rev. Drug Discov. 12, 371-387 (2013).

8. Butler, M. S., Blaskovich, M. A. \& Cooper, M. A. Antibiotics in the clinical pipeline at the end of 2015. J. Antibiot. 70, 3-24 (2017).

9. Cassell, G. H. \& Mekalanos, J. Development of antimicrobial agents in the era of new and reemerging infectious diseases and increasing antibiotic resistance. JAMA 285, 601-605 (2001).

10. Coates, A. R. M., Halls, G. \& Hu, Y. Novel classes of antibiotics or more of the same? Br. J. Pharmacol. 163, 184-194 (2011).

11. Lehar, S. M. et al. Novel antibody-antibiotic conjugate eliminates intracellular S. aureus. Nature 527, 323-328 (2015).

12. Seiple, I. B. et al. A platform for the discovery of new macrolide antibiotics. Nature 533, 338-345 (2016)

13. Nikaido, H. Antibiotic resistance caused by gram-negative multidrug efflux pumps. Clin. Infect. Dis. 27(Suppl 1), S32-S41 (1998).

14. Navarro, L. et al. A plant miRNA contributes to antibacterial resistance by repressing auxin signaling. Science 312, 436-439 (2006).

15. Alekshun, M. N. \& Levy, S. B. Molecular mechanisms of antibacterial multidrug resistance. Cell 128, 1037-1050 (2007).

16. Peschel, A. \& Sahl, H.-G. The co-evolution of host cationic antimicrobial peptides and microbial resistance. Nat. Rev. Microbiol. 4, 529-536 (2006).

17. Ling, L. L. et al. A new antibiotic kills pathogens without detectable resistance. Nature 517, 455-459 (2015)

18. Wilson, M. C. et al. An environmental bacterial taxon with a large and distinct metabolic repertoire. Nature 506, 58-62 (2014)

19. Rutledge, P. J. \& Challis, G. L. Discovery of microbial natural products by activation of silent biosynthetic gene clusters. Nat. Rev. Microbiol. 13, 509-523 (2015).

20. Zipperer, A. et al. Human commensals producing a novel antibiotic impair pathogen colonization. Nature 535, 511-516 (2016).

21. Aminov, R. I. A brief history of the antibiotic era: lessons learned and challenges for the future. Front. Microbiol. 1, 134 (2010).

22. Fischbach, M. A. \& Walsh, C. T. Antibiotics for emerging pathogens. Science 325, 1089-1093 (2009).

23. Bax, B. D. et al. Type IIA topoisomerase inhibition by a new class of antibacterial agents. Nature 19, 935-940 (2010).

24. Zhao, Q., Wang, M., Xu, D., Zhang, Q. \& Liu, W. Metabolic coupling of two small-molecule thiols programs the biosynthesis of lincomycin A. Nature $\mathbf{5 1 8}$, 115-119 (2015).

25. Tommasi, R., Brown, D. G., Walkup, G. K., Manchester, J. I. \& Miller, A. A. ESKAPEing the labyrinth of antibacterial discovery. Nat. Rev. Drug. Discov. 14, 529-542 (2015)

26. Finney, N. S. Combinatorial discovery of fluorophores and fluorescent probes. Curr. Opin. Chem. Biol. 10, 238-245 (2006).

27. Lee, J. S., Lee, J. W., Kang, N., Ha, H. H. \& Chang, Y. T. Diversity-oriented approach for chemical biology. Chem. Rec. 15, 495-510 (2015).

28. Vendrell, M., Zhai, D., Er, J. C. \& Chang, Y. T. Combinatorial strategies in fluorescent probe development. Chem. Rev. 112, 4391-4420 (2012).

29. Yun, S. W. et al. Diversity oriented fluorescence library approach (DOFLA) for live cell imaging probe development. Acc. Chem. Res. 47, 1277-1286 (2014). 
30. Alamudi, S. H. et al. Development of background-free tame fluorescent probes for intracellular live cell imaging. Nat. Commun. 7, 11964 (2016).

31. Kang, N. Y., Ha, H. H., Yun, S. W., Yu, Y. H. \& Chang, Y. T. Diversity-driven chemical probe development for biomolecules: beyond hypothesis-driven approach. Chem. Soc. Rev. 40, 3613-3626 (2011).

32. Lim, S. H. et al. Rosamines targeting the cancer oxidative phosphorylation pathway. PloS ONE 9, e82934 (2014).

33. Wu, L. \& Burgess, K. Synthesis and spectroscopic properties of rosamines with cyclic amine substituents. J. Org. Chem. 73, 8711-8718 (2008).

34. Baeyer, A. Uber eine neue Klasse von Farbstoffen (On a new class of dyes). Ber. Dtsch. Chem. Ges. 4, 555-558 (1871).

35. Jiao, G.-S., Castro, J. C., Thoresen, L. H. \& Burgess, K. Microwave-assisted syntheses of regioisomerically pure bromorhodamine derivatives. Org. Lett. 5, 3675-3677 (2003).

36. Minta, A., Kao, J. P. \& Tsien, R. Y. Fluorescent indicators for cytosolic calcium based on rhodamine and fluorescein chromophores. J. Biol. Chem. 264 8171-8178 (1989).

37. Ahn, Y. H., Lee, J. S. \& Chang, Y. T. Combinatorial rosamine library and application to in vivo glutathione probe. J. Am. Chem. Soc. 129, 4510-4511 (2007).

38. Bajorath, J. Selected concepts and investigations in compound classification, molecular descriptor analysis, and virtual screening. J. Chem. Inf. Comput. Sci. 41, 233-245 (2001).

39. Rogers, D. \& Hahn, M. Extended-connectivity fingerprints. J. Chem. Inf. Model. 50, 742-754 (2010).

40. Hassan, M., Brown, R. D., Varma-O'brien, S. \& Rogers, D. Cheminformatics analysis and learning in a data pipelining environment. Mol. Divers. 10, 283-299 (2006).

41. Yang, Y. J. et al. A convient preparation of xanthene dyes. J. Org. Chem. 70, 6907-6912 (2005).

42. Yang, Y. J. et al. Preparation of novel rhodamine dyes useful as antibacterial agents. CN Patent Application CN108570032A, filed on Mar. 9th, 2017.

43. Grimm, J. B., Brown, T. A., Tkachuk, A. N. \& Lavis, L. D. General synthetic method for Si-fluoresceins and Si-rhodamines. ACS Cent. Sci. 3, 975-985 (2017).

44. Fischer, C. \& Sparr, C. Direct transformation of esters into heterocyclic fluorophores. Angew. Chem. Int. Ed. 57, 2436-2440 (2018).

45. Best, Q. A., Xu, R., McCarroll, M. E., Wang, L. \& Dyer, D. J. Design and investigation of a series of rhodamine-based fluorescent probes for optical measurements of pH. Org. Lett. 12, 3219-3221 (2010).

46. Wu, X., Li, Z., Yang, L., Han, J. \& Han, S. A self-referenced nanodosimeter for reaction based ratiometric imaging of hypochlorous acid in living cells. Chem. Sci. 4, 460-467 (2013).

47. Mei, J., Leung, N. L. C., Kwok, R. T. K., Lam, J. W. Y. \& Tang, B. Z. Aggregation-induced emission: together we shine, united we soar! Chem. Rev. 115, 11718-11940 (2015).

48. Marks, K. M. \& Nolan, G. P. Chemical labeling strategies for cell biology. Nat. Methods 3, 591-596 (2006).

49. Qian, Y. et al. Selective fluorescent probes for live-cell monitoring of sulphide. Nat. Commun. 2, 495 (2011).

50. Garner, A. L. et al. Specific fluorogenic probes for ozone in biological and atmospheric samples. Nat. Chem. 1, 316-321 (2009).

51. Bamfield, P. \& Hutchings, M. G. Chromic Phenomena, Technological Applications of Coulour Chemistry. ${ }^{\text {nd }}$ Ed. (RSC Publishing: London, 2010).

52. WHO (2017) Global priority list of antibiotic-resistant bacteria to guide research, discovery, and development of new antibiotics. http://www.who.int/ medicines/publications/global-priority-list-antibiotic-resistant-bacteria/en/ February 27, 2017.

53. Pankey, G. A. \& Sabath, L. D. Clinical relevance of bacteriostatic versus bactericidal mechanisms of action in the treatment of Gram-positive bacterial infections. Clin. Infect. Dis. 38, 864-870 (2004).
54. Fernández, L., Breidenstein, E. B. \& Hancock, R. E. Creeping baselines and adaptive resistance to antibiotics. Drug Resist. Updates 14, 1-21 (2011).

55. Amato, S. M., Orman, M. A. \& Brynildsen, M. P. Metabolic control of persister formation in Escherichia coli. Mol. Cell 50, 475-487 (2013).

56. Allison, K. R., Brynildsen, M. P. \& Collins, J. J. Metabolite-enabled eradication of bacterial persisters by aminoglycosides. Nature 473, 216-220 (2011).

57. Sparling, P. F. Kasugamycin resistance: 30 S ribosomal mutation with an unusual location on the Escherichia coli chromosome. Science 167, 56-58 (1970).

58. Basturea, G. N., Dague, D. R., Deutscher, M. P. \& Rudd, K. E. YhiQ is RsmJ, the methyltransferase responsible for methylation of G1516 in 16S rRNA of $E$. coli. J. Mol. Bio. 415, 16-21 (2012).

\section{Acknowledgements}

This work is supported by the Fundamental Research Funds for the Central Universities (No. WY1516017) and the National Natural Science Foundation of China (Nos. 21572061, 21822805, and 81573329).

\section{Author contributions}

Y.Y., D.C. and X.Q. conceived the projects. X.L. designed the synthetic method, synthesis RD1-72 and acquired the UV-Vis absorption and fluorescence properties. Y.X., L.G., Z.L. and T.H. consynthesized RD73-74 and C1-C2. L.Q. measured the MIC's of standardized bacteria, collected the dose-kill curves and carried out the induced resistance test and did the SEM imaging of bacteria. L.S., X.D., J.B. and J.W. contributed to phenotypic screenings. X.L., Y.T., Y.Z., J.Z. and W.Z. did the confocal imaging of mammalian cells. X.W. and H.L. calculated the diversity index. F.H. measured the MIC's of the clinical strains. All authors contributed to manuscript preparations.

\section{Additional information}

Supplementary Information accompanies this paper at https://doi.org/10.1038/s41467018-08241-3.

Competing interests: All authors declare no competing interests.

Reprints and permission information is available online at http://npg.nature.com/ reprintsandpermissions/

Journal peer review information: Nature Communications thanks the anonymous reviewers for their contribution to the peer review of this work. Peer reviewer reports are available.

Publisher's note: Springer Nature remains neutral with regard to jurisdictional claims in published maps and institutional affiliations.

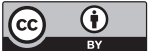

Open Access This article is licensed under a Creative Commons Attribution 4.0 International License, which permits use, sharing, adaptation, distribution and reproduction in any medium or format, as long as you give appropriate credit to the original author(s) and the source, provide a link to the Creative Commons license, and indicate if changes were made. The images or other third party material in this article are included in the article's Creative Commons license, unless indicated otherwise in a credit line to the material. If material is not included in the article's Creative Commons license and your intended use is not permitted by statutory regulation or exceeds the permitted use, you will need to obtain permission directly from the copyright holder. To view a copy of this license, visit http://creativecommons.org/ licenses/by/4.0/.

(C) The Author(s) 2019 